\title{
High-frequency large-area surveys of extragalactic sources and their relevance for CMB experiments
}

\section{Ronald D. Ekers ${ }^{*}$}

Australia Telescope National Facility

CSIRO, P.O. Box 76, Epping, NSW 1710, Australia

E-mail: Ron.Ekersecsiro.au

\section{Elaine M. Sadler}

School of Physics, University of Sydney,

NSW 2006, Australia

E-mail: ems aphysics.usyd.edu.au

\section{Roberto Ricci}

Australia Telescope National Facility,

CSIRO, P.O. Box 76, Epping, NSW 1710, Australia

E-mail: Roberto.Ricciecsiro.au

\begin{abstract}
We discuss the nature of the discrete extragalactic foreground sources at frequencies between 20 and $100 \mathrm{GHz}$, and their impact on $\mathrm{CMB}$ measurements. High frequency radio surveys are essential to establish the sample statistics of this class of sources which are strongly underrepresented in the existing catalogues at lower frequencies. The difficulties in making higher frequency surveys are discussed and we describe the new $20 \mathrm{GHz}$ blind survey with the Australia Telescope Compact Array (AT20G). In addition to characterising the population statistics we can make a real source catalogue which could be used to remove this component of the foreground confusion. Some of our first results which may be relevant to Planck are presented. Note that most of the AT20G data is in an early stage of data reduction and most is not yet published.
\end{abstract}

CMB and Physics of the Early Universe - International Conference

Ischia, Italy

April $20-22,2006$

\footnotetext{
${ }^{*}$ Speaker
} 


\section{Introduction}

\subsection{Extragalactic Radio Sources}

Most of our knowledge of extragalactic radio sources is based on surveys at frequencies much lower than those used by Planck. These surveys are dominated by powerful sources with steep spectrum optically thin synchrotron radiating lobes (Fig 1). The flatter spectrum cores in the nuclei of these radio galaxies and QSOs which will dominate the Planck foreground are a minor component of the low frequency surveys. For example, the NVSS survey at $1.4 \mathrm{GHz}$ has $63 \%$ lobe dominated sources and only $37 \%$ core dominated sources. However, the AT20G survey at $20 \mathrm{GHz}$ has $87 \%$ cores and only $13 \%$ lobes.

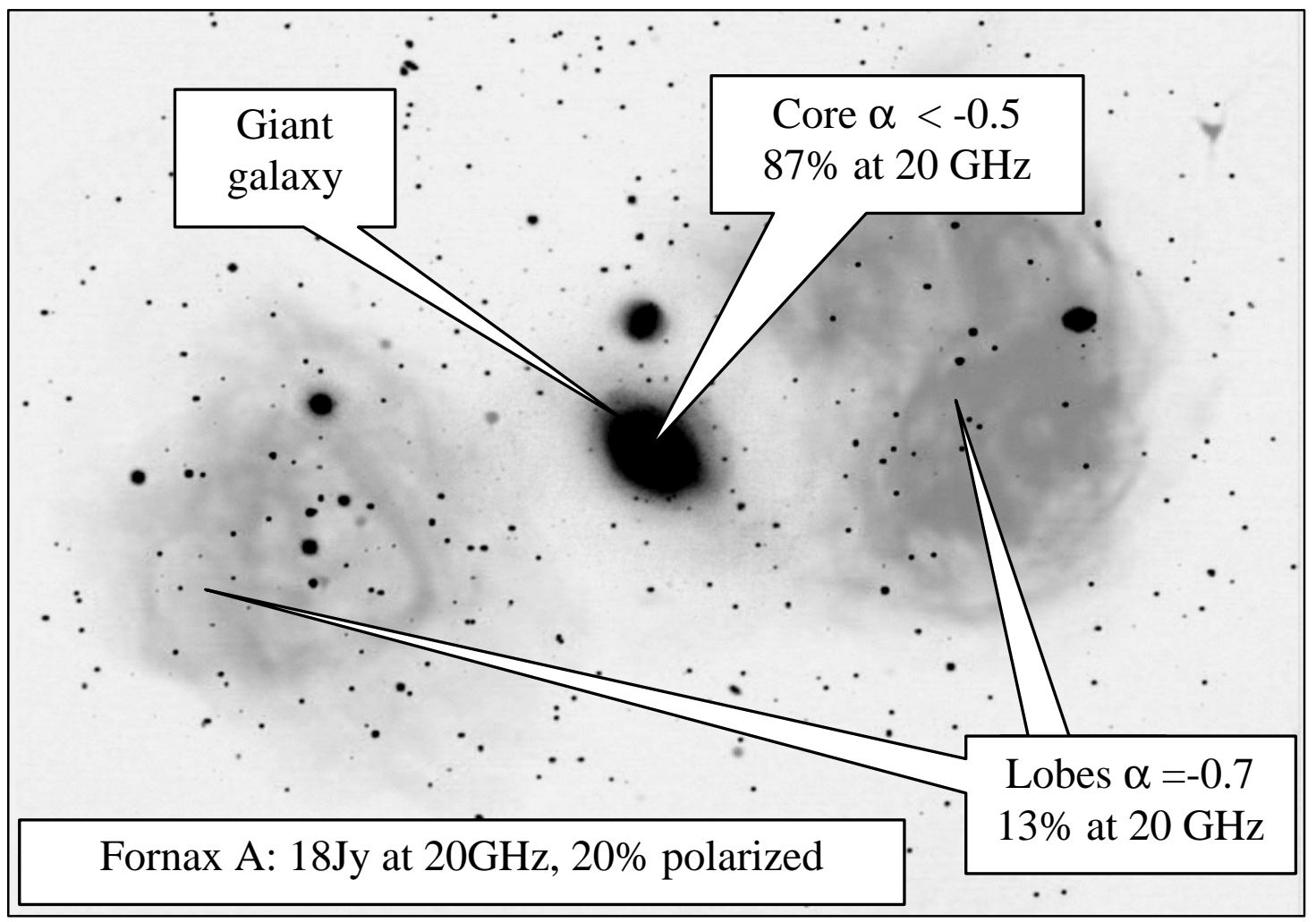

Figure 1: Radio Galaxy Fornax A [1]

\subsection{Surveys at High Frequency}

Most radio surveys have been conducted at lower radio frequencies for good reasons. For a telescope with diffraction limited field of view, the number of pointings needed for a given survey area scales as $v^{2}$. The radio flux, $S$, is $\propto v^{-0.7}$ for typical radio sources. For a given receiver noise the time needed per pointing to reach a flux level, $S$, is $\propto \mathrm{S}^{-2}$, and hence the survey time scales as $\propto v^{3.4}$. This means that a $1.2 \mathrm{~cm}$ survey takes 240 times as long as a $6 \mathrm{~cm}$ survey for the same area and source density. 
Table 1: Previous high frequency surveys

\begin{tabular}{|l|r|r|r|}
\hline $\begin{array}{l}\text { Project } \\
\text { Frequency }\end{array}$ & $\begin{array}{r}\text { Number of } \\
\text { Sources }\end{array}$ & $\begin{array}{r}\text { Area } \\
\text { (sq. deg.) }\end{array}$ & $\begin{array}{r}\text { Flux Limit } \\
(\mathrm{mJy})\end{array}$ \\
\hline $\begin{array}{l}\text { Taylor et al.2001 [2] } \\
15 \mathrm{GHz}\end{array}$ & 66 & 60 & 20 \\
\hline $\begin{array}{l}\text { Waldram et al. 2003 (9C) [3] } \\
15(33) \mathrm{GHz}\end{array}$ & 465 & 520 & 25 \\
\hline $\begin{array}{l}\text { Hinshaw et al. 2006 (WMAP) [4] } \\
23 \mathrm{GHz}\end{array}$ & 323 & All sky & 1000 \\
\hline $\begin{array}{l}\text { Ricci et al. 2004 [5] } \\
18 \mathrm{GHz}\end{array}$ & 126 & 1216 & 100 \\
\hline $\begin{array}{l}\text { AT20G } \\
20 \mathrm{GHz}\end{array}$ & 4146 & $\begin{array}{r}10,000 \\
25 \% \text { sky }\end{array}$ & 50 \\
\hline
\end{tabular}

\section{The ATCA 20 GHz survey: AT20G}

The Australia Telescope Compact Array (ATCA) is currently being used to carry out a radio continuum survey of the entire southern sky at $20 \mathrm{GHz}$ (AT20G) [5]. The wide bandwidth of an analogue correlator [6], originally developed for the Taiwanese CMB instrument AMiBA [7], combined with the fast scanning speed of the ATCA, makes it possible to scan large areas of sky at high sensitivity despite the small $(2.3 \mathrm{arcmin})$ primary beam width at $20 \mathrm{GHz}$. The survey frequency is $16-24 \mathrm{GHz}$, with bandwidth of $8 \mathrm{GHz}$ divided into 8 frequency channels. The survey flux limit is about $50 \mathrm{mJy}$; the survey will be complete above $70 \mathrm{mJy}$.

The fast-scanning survey measures approximate positions and flux densities for all candidate sources above the detection threshold. Follow-up $20 \mathrm{GHz}$ imaging of these candidate detections is then carried out a few weeks later, using the ATCA and its standard digital correlator. These follow-up images allow us to confirm detections, and to measure accurate positions, flux densities and polarization for the detected sources. Finally, the confirmed sources are also imaged at 5 and $8 \mathrm{GHz}$ to measure their radio spectra, polarisation and angular size. The pilot survey using this technique has been published [5] and more details of the ongoing main survey are included in [8].

\subsection{AT20G survey progress}

- Declination $-60^{\circ}$ to $-70^{\circ}$ pilot survey (2002) [5]

1 baseline, $3.4 \mathrm{GHz}$ bandwidth. Incomplete sky coverage

This pilot survey database has been used for most of the data presented here.

- Declination $-60^{\circ}$ to $-70^{\circ}$; full test (2003)

3 baselines, $3 \mathrm{GHz}$ bandwidth

300 sources (120 Galactic) soft completeness limit of about $100 \mathrm{mJy}$ 
- Declination $-30^{\circ}$ to $-50^{\circ}$ main survey (2004)

3 baselines, $7 \mathrm{GHz}$, calibrated

1420 sources down to $40 \mathrm{mJy}$ ( 170 Galactic)

- Declination $-50^{\circ}$ to $-90^{\circ}$ main survey (2005)

1777 sources down to $40 \mathrm{mJy}$ ( 520 Galactic)

in 2005 we also repeated the declination $-60^{\circ}$ to $-70^{\circ}$ strip for the 3rd time and this has been used for the variability analysis [8]

- Declination $-30^{\circ}$ to $-15^{\circ}$ main survey (2006) scheduled August 2006

\subsection{Sky maps of the AT20G confirmed sources from the main survey (2004-2005)}
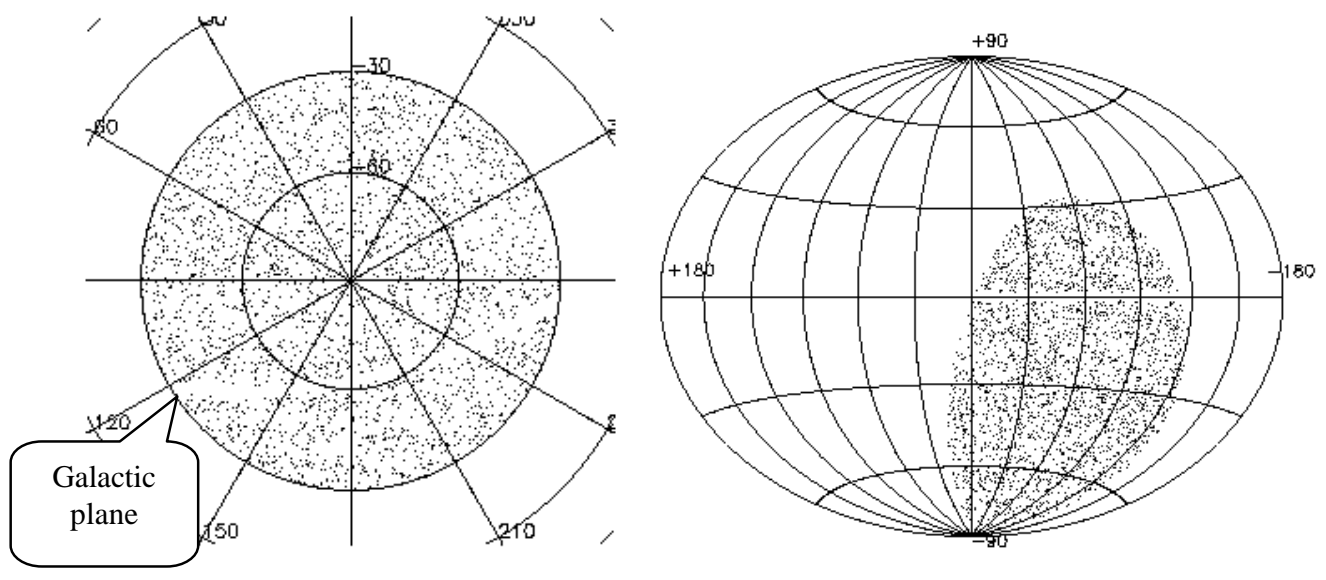

Figure.2 (a) Polar projection in celestial coordinates; (b) Aitoff projection in galactic coordinates

2,800 extragalactic sources detected in 10300 sq deg. Galactic Plane sources not included.

\subsection{Completeness and Reliability}

From the comparison with SUMSS and NVSS and analysis of the fraction of $20 \mathrm{GHz}$ sources confirmed in the follow up, we expect very high completeness to $70 \mathrm{mJy}$. The 4146 extragalactic sources detected (declination $-30^{\circ}$ to $-90^{\circ}$ ) matches the predicted source density [9]. Between 40 and $70 \mathrm{mJy}$ there will be some variability in completeness for different regions of the sky due to variable observing conditions. Since the AT20G Catalogue includes follow up observations for all candidate sources, the reliability is essentially $100 \%$. 


\subsection{Survey Source Density v Galactic Latitude}

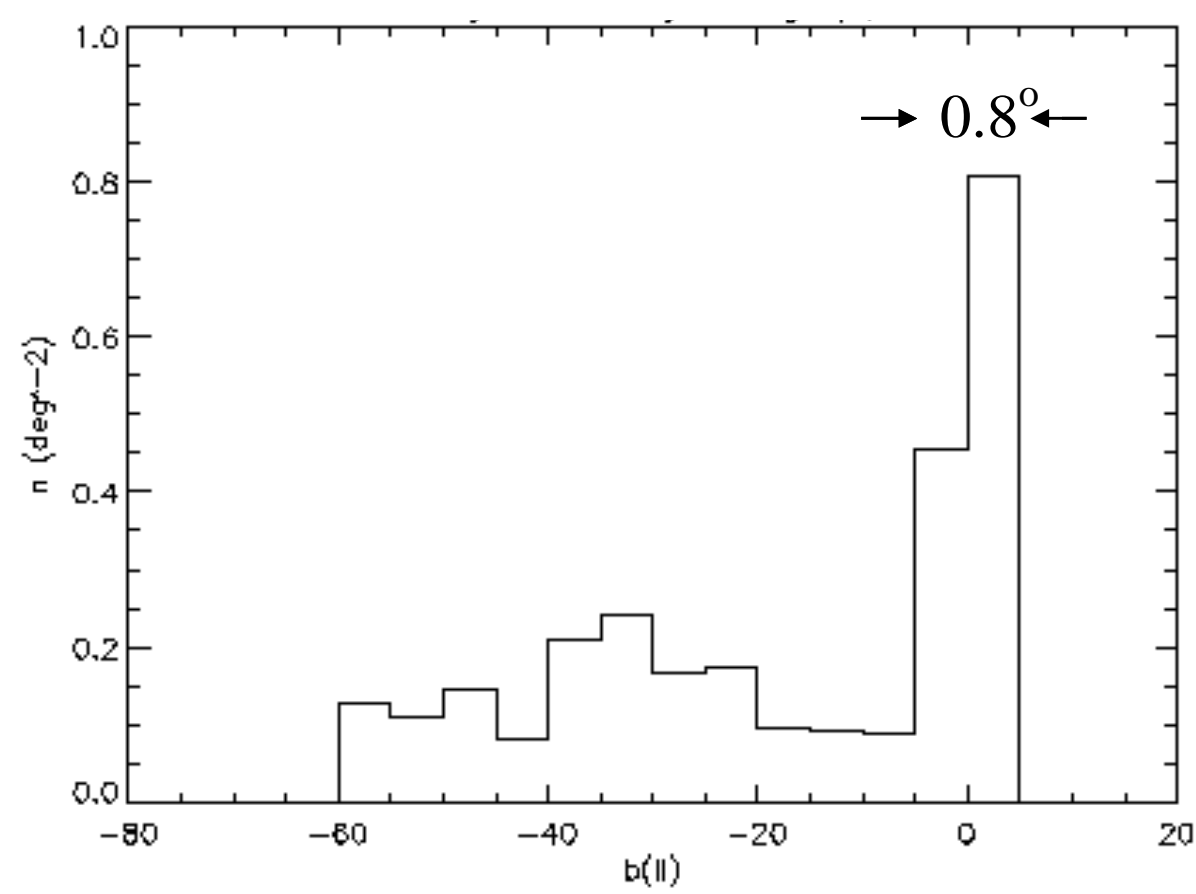

Figure 3. Histogram of source density in 1-degree bins of galactic latitude $b$ for the pilot study (declination $-60^{\circ}$ to $-70^{\circ}$ ). This zone only includes the Galactic latitudes from $b=-60^{\circ}$ to $+3^{\circ}$. [5]. The peak near $b=0^{\circ}$ corresponds to Galactic Disc sources such as HII regions.

\subsection{Differential Source Counts}

Using the pilot survey corrected for sky coverage and excluding the Galactic Plane we have extragalactic sources $\mathrm{N}(\mathrm{S}) \propto \mathrm{S}^{-2.0 \pm 0.2}$ for $\mathrm{S}_{18 \mathrm{GHz}}>100 \mathrm{mJy}$. Our counts are in good agreement with those by Waldram et al [3] at $15 \mathrm{GHz}$ and WMAP counts [10].

\section{3. $20 \mathrm{GHz}$ source populations}

\subsection{Polarization}

The polarization properties of sources which are strong at high frequencies are very poorly known [11] yet these sources will be the major impediment to detecting CMB polarization anisotropies. In the AT20G all four Stokes parameters are measured at 20, 8 , and $5 \mathrm{GHz}$ for all sources. The distribution of fractional polarization and polarized flux at $20 \mathrm{GHz}$ is shown in Figures $4 \mathrm{a}$ and $4 \mathrm{~b}$ for sources from the declination zone $-30^{\circ}$ to $-50^{\circ}$. The median fractional polarisation is $2.6 \%$, somewhat lower than that reported in [12]. 


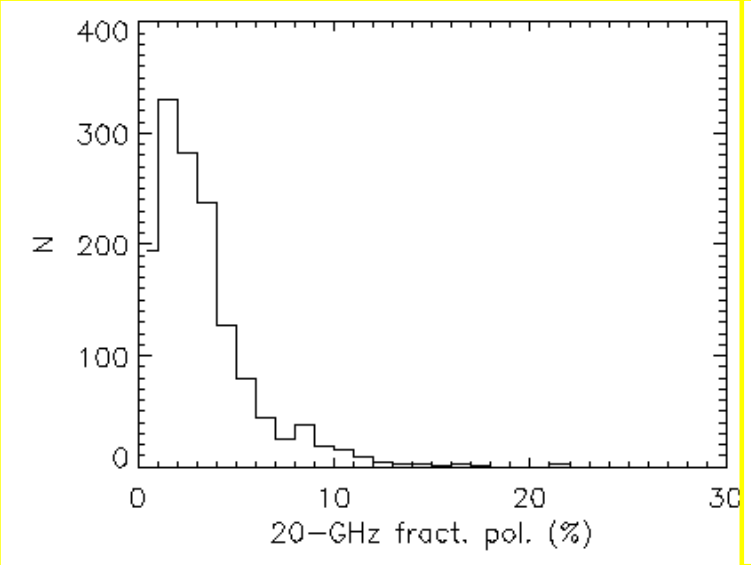

(a)

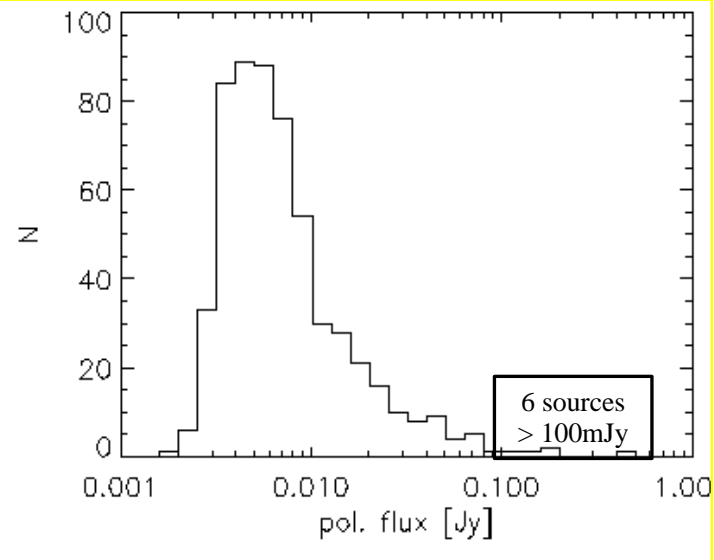

(b)

Figure 4. Polarization of AT20G sources in the declination zone $-30^{\circ}$ to $-50^{\circ}$. (a) Fractional polarised flux distribution; (b) Polarised flux distribution.

\subsection{Radio Spectra of the AT20G sources}

Figure 5 shows some representative radio spectra for sources in our sample. It is clear we see a wide variety of spectral shapes, most of which cannot be fitted by a single power-law over the frequency range 1-20 GHz. We can distinguish four main kinds of spectra which are illustrated in Fig. 5.

(i) Sources with steep (falling) spectra over the whole range $843 \mathrm{MHz}$ to $20 \mathrm{GHz}$ (e.g. J04086545).

(ii) Sources with peaked (GPS) spectra, in which the flux density rises at low frequency and falls at high frequency (e.g. J0201-6638).

(iii) Sources with inverted (rising) radio spectra over the whole frequency range (e.g. J01136753).

(iv) Sources with an upturn in their spectrum, where the flux density is falling at lower frequencies, but then turns up and begins to rise above 5-8 GHz (e.g. J2213-6330).

In addition, a small number of sources have a radio spectra in which the flux density is essentially constant over the entire frequency range observed (e.g. J0220-6330).

At centimetre wavelengths the radio emission from flat-spectrum $\left(\alpha>-0.5 ; S \propto v^{\alpha)}\right.$ objects is dominated by a compact, self-absorbed component, while steep-spectrum objects $(\alpha<-0.5)$ are dominated by optically-thin synchrotron emission. The flat- and steep-spectrum populations are usually considered separately when modelling the cosmic evolution of radio sources [13].

As pointed out by Peacock [13] the radio spectral index is only valid as a diagnostic tool if it is measured over a frequency interval small enough that the effects of spectral curvature can be neglected. Because many of the sources in our sample have significant spectra curvature over the frequency range 1-20 GHz, we therefore use a 'radio two-colour' diagram, rather than a single spectral index, to characterize the high-frequency radio-source population. Fig. 6 compares a low-frequency spectral index $\alpha_{\mathrm{L}}$ (which corresponds closely to the spectral index 
traditionally used to separate flat spectrum and steep spectrum radio sources) with a highfrequency spectral index $\alpha_{\mathrm{H}}$ which measures the spectral shape above $8 \mathrm{GHz}$.
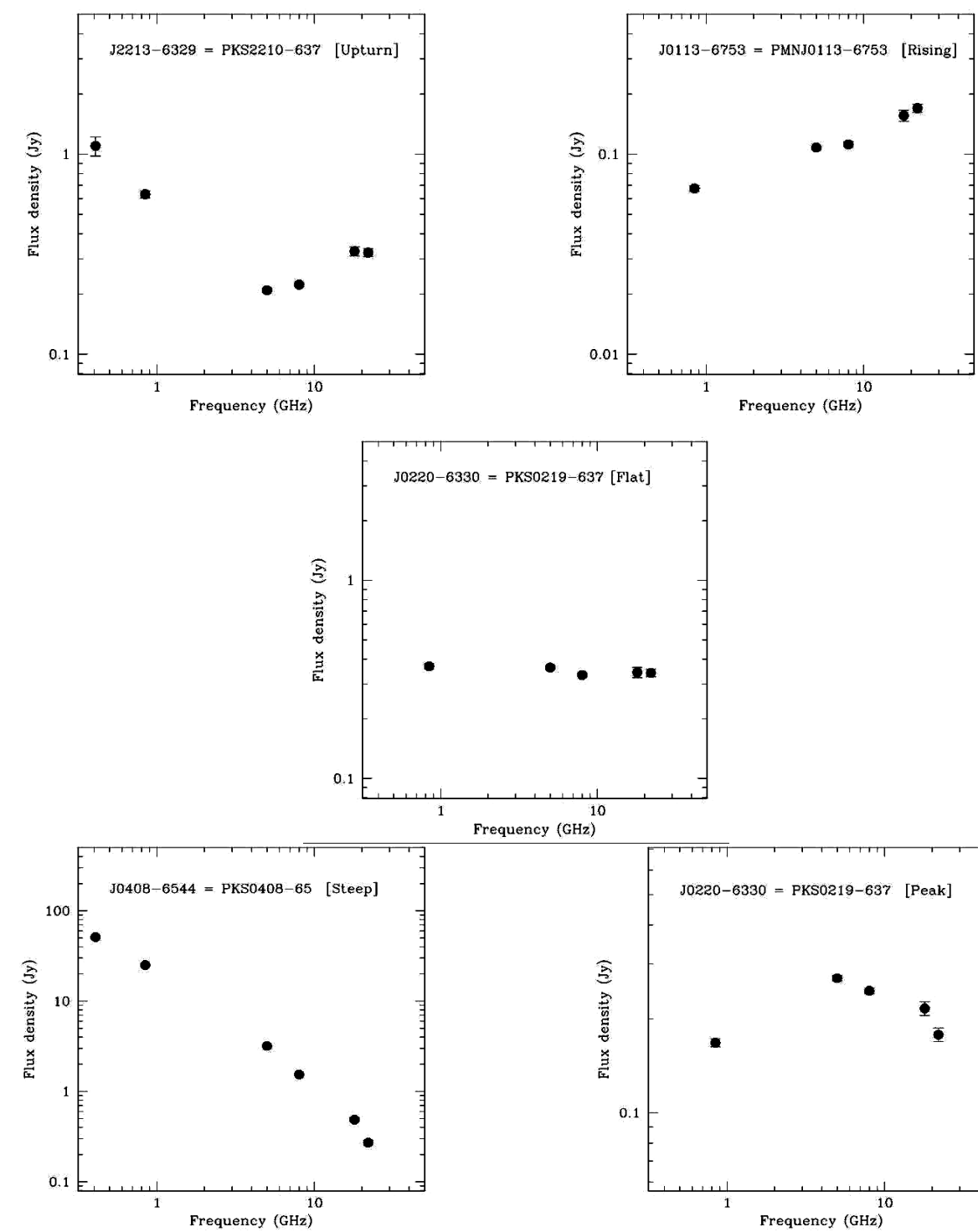

20-6330 $=$ PKS0219-637 [Flat $]$

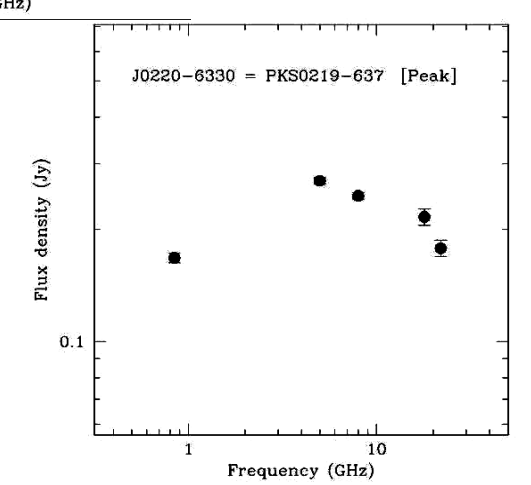

Figure 5. Examples of radio spectra for each of the four spectral classes identified in the text (Upturn, Rising, Steep and Peak), together with a spectrum classified as Flat $(\alpha<0.1$ for both 0.84-5 GHz and 8-20 GHz). 


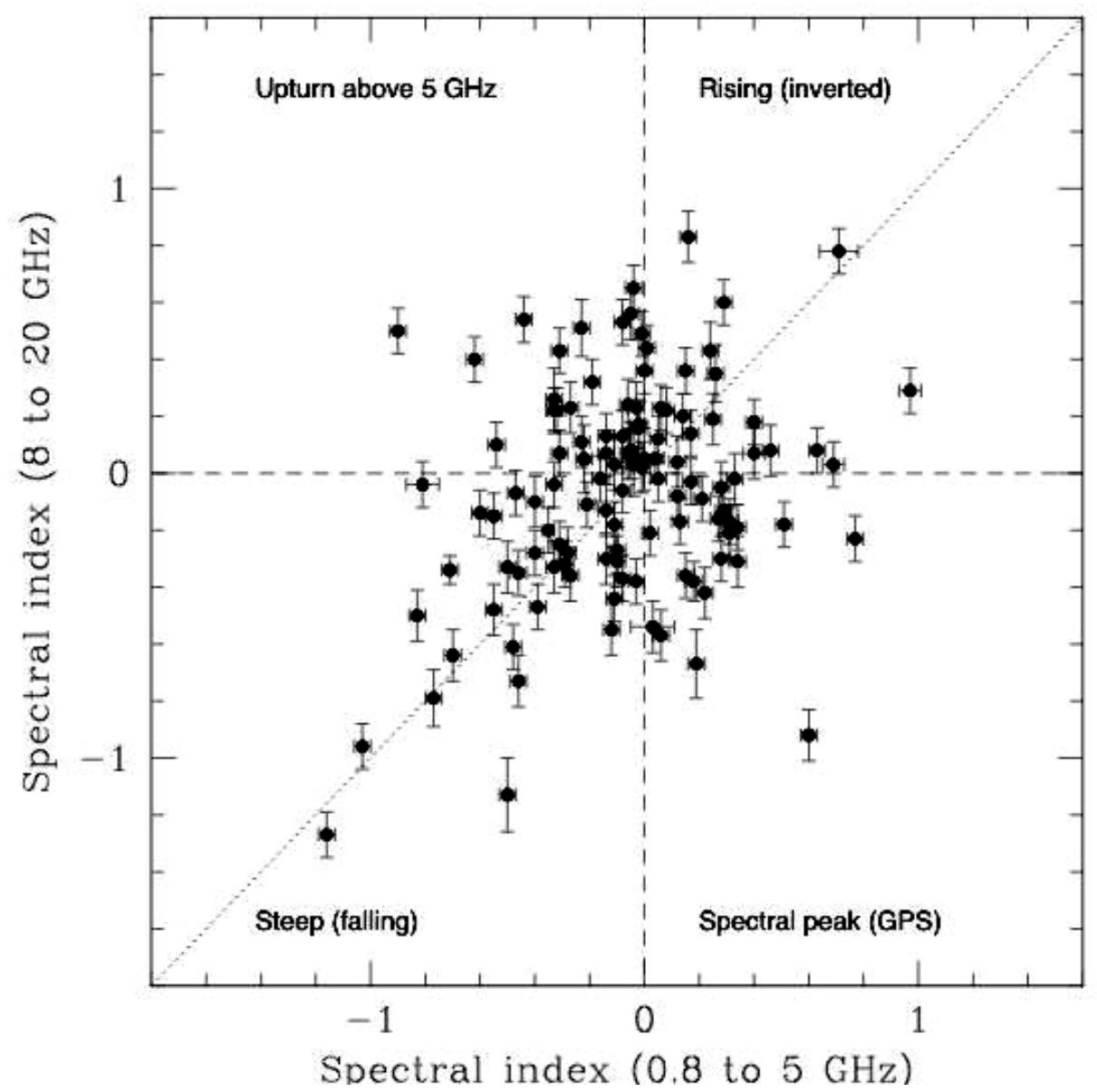

Figure 6. Radio 'two-colour diagram' for 119 extragalactic sources in which have good-quality multi-frequency observations made in late 2003 [8].

Table 2. Distribution of our sample in the five spectral classes

\begin{tabular}{|l|l|r|l|}
\hline Spectrum & How defined & Number & Fraction \\
\hline Steep & $\alpha_{\mathrm{L}}<0, \alpha_{\mathrm{H}}<0$ & 32 & $32 \pm 6 \%$ \\
\hline Upturn & $\alpha_{\mathrm{L}}<0, \alpha_{\mathrm{H}}>0$ & 22 & $22 \pm 5 \%$ \\
\hline Rising (Inv.) & $\alpha_{\mathrm{L}}>0, \alpha_{\mathrm{H}}>0$ & 18 & $18 \pm 4 \%$ \\
\hline Peak & $\alpha_{\mathrm{L}}>0, \alpha_{\mathrm{H}}<0$ & 23 & $23 \pm 4 \%$ \\
\hline Flat & $-0.1<\left(\alpha_{\mathrm{L}}, \alpha_{\mathrm{H}}\right)<0.1$ & 6 & $6 \pm 2 \%$ \\
\hline Total & \multicolumn{3}{|l}{} \\
\hline
\end{tabular}

The dotted line shows the relation for galaxies whose spectra follow a single power-law from 0.8 to $18 \mathrm{GHz}$. It is clear that only a small fraction of sources fall on or near the dotted line, and that $\alpha_{\mathrm{L}}$ and $\alpha_{\mathrm{H}}$ are not strongly correlated. Over 30\% of the sources in Fig. 6 have flat or inverted spectra between 8 and $20 \mathrm{GHz}$ (i.e. $\alpha_{\mathrm{H}}>0$ ), and more than half of these (like J22136330 in Fig. 6) have steep radio spectra below $5 \mathrm{GHz}$ and would not have been predicted as 
strong $20 \mathrm{GHz}$ sources on the basis of their low frequency spectra. In contrast, many sources with flat or inverted spectra below $5 \mathrm{GHz}$ turn over and become steep above $8 \mathrm{GHz}$.

\section{3. $20 \mathrm{GHz}$ v $90 \mathrm{GHz}$ fluxes}

Pilot observations were made in July 2004 using the new ATCA 3mm receiver.

57 objects with flat or rising spectrum were observed at $93 \mathrm{GHz}$ and re-observed at $18 \mathrm{GHz}$ to avoid any effects of variability. 51 sources were detected; only one source has $90 \mathrm{GHz}$ flux greater than that expected from a power law extrapolation. The median spectral index between 20 and $93 \mathrm{GHz}$ is $\alpha=-0.24\left(\mathrm{~S} \propto v^{\alpha}\right)$.

\subsection{Variability at $20 \mathrm{GHz}$}

There have been only a few studies of radio source variability at frequencies above $5 \mathrm{GHz}$ and these studies are based largely on objects pre-selected at lower frequencies, so may not give a complete picture of the variability of the high frequency source population as a whole. Owen, Spangler \& Cotton [14] investigated the variability of a sample of strong $\left(\mathrm{S}_{90 \mathrm{GHz}}>1 \mathrm{Jy}\right)$ flat spectrum sources at 5 and $90 \mathrm{GHz}$ over a one year period and they found that these sources were only slightly more variable at $90 \mathrm{GHz}$ than at $5 \mathrm{GHz}$. Tingay et al. [15] also found that the level of variability of strong, compact radio sources increased only moderately with frequency. However, a multi frequency $(5,8,15,22,37 \mathrm{GHz})$ analysis [16] based on UMRAO and Metsühovi catalogues did show an increase in variability index with increasing frequency.

In order to explore the variability in a sample selected at high frequency we have remeasured the $20 \mathrm{GHz}$ flux densities of the AT20G sources from the pilot survey [8] every year since 2002. We have analysed in detail flux changes for 121 sources between 2003 and 2004. For those observations, variability of $6 \%$ or more over this one-year interval is detectable for all sources in this data set. Table 2 shows the distribution of the de-biased variability index [17] for these sources [8]. Our median variability index over one year is $6.9 \%$ and only $5 / 121$ sources varied by more than $30 \%$. These results are fully consistent with the results from samples selected at lower frequency.

Table 2. : Variability Index at $20 \mathrm{GHz}$

\begin{tabular}{|l|r|l|}
\hline Variability & number & Faction \\
\hline$<10 \%$ & 63 & $58 \pm 7 \%$ \\
\hline $10-20 \%$ & 9 & $27 \pm 5 \%$ \\
\hline $20-30 \%$ & 1 & $10 \pm 3 \%$ \\
\hline$>30 \% \quad$ Total & 5 & $5 \pm 2 \%$ \\
\hline \multicolumn{2}{|r|}{} \\
\hline
\end{tabular}

In addition to monitoring a given sample of sources we have now re-surveyed the same -$60^{\circ}$ to $-70^{\circ}$ declination strip three times $(2002,2003,2005)$ and we can also compare with the independent WMAP survey. WMAP detected all except one of our $\mathrm{S}_{20}>1$ Jy sources. The sole exception being the strongly variable Seyfert galaxy 0623-6436. The recovery rate for sources in our independent surveys $\left(S_{20}>150 \mathrm{mJy}\right)$ is $95 \%$. These results suggest that catalogues will 
be robust on time scales of a few years. We see no new population of highly variable sources and our data is consistent with the observation that variability is characterised by a burst of short term variability followed by a long period of quiescence [18].

\section{Future observations}

In 2006 we will continue the survey north from declination $-30^{\circ}$ to $-15^{\mathrm{o}}$. We will use the ATCA to measure fluxes of the stronger sources at $100 \mathrm{GHz}$. By mid 2007 the ATCA will also be equipped to measure polarization at $100 \mathrm{GHz}$ and with receivers covering the $30-50 \mathrm{GHz}$ band.

Other telescopes will also have new capabilities. The GBT is being equipped with a 90 $\mathrm{GHz}$ focal plane array and very high frequency surveys will be possible in the northern hemisphere. The EVLA will have much higher sensitivity at $20-50 \mathrm{GHz}$ and more flexible scanning modes. ALMA will become the dominant telescope to measure fluxes and polarization for a wide range of frequencies $>90 \mathrm{GHz}$.

\section{Predicting high frequency source samples from lower frequency catalogues}

We have made a preliminary comparison in the declination zone $-30^{\circ}$ to $-40^{\circ}$. We used the NVSS $(1.4 \mathrm{GHz})$ and SUMMS $(0.8 \mathrm{GHz})$ catalogues to predict fluxes at $20 \mathrm{GHz}$. We predicted 559 sources $>50 \mathrm{mJy}$ at $20 \mathrm{GHz}$ but only 175 were detected at this level. If we check how many AT20G sources are in NVSS at any flux level, we find $100 \%$ for $\mathrm{S}_{20 \mathrm{GHz}}>100 \mathrm{mJy}$, decreasing to $83 \%$ for $\mathrm{S}_{20 \mathrm{GHz}}>40 \mathrm{mJy}$. So most of the high frequency sources which will impact Planck are in NVSS, but they are a small fraction of the total NVSS sample and one cannot predict which NVSS sources will have up-turning spectra.

\section{Comments and Questions re Planck foreground sources}

- South of declination $-15^{\circ}$, current telescopes could measure and remove every significant confusing source in the Planck low frequency foreground. North of declination $-15^{\circ}$, which is the current planned limit of AT20G, we would need to extend the survey and this would have to be justified based on the value of the survey extension to Planck.

- It would be possible to make follow-up observations during Planck mission to avoid the effects of variability. Eg the ATCA can measure 500 sources per day at 20 and $40 \mathrm{GHz}$, and could measure $100 \mathrm{GHz}$ fluxes for a smaller subset. In the north, the VLA could make similar observations if we could identify the sources to observe.

- A simultaneous blind survey would even be possible, but would be very time consuming. Is there a strong enough case to do this?

- Should we do a deeper survey in a smaller area? Eg the south celestial or equatorial pole. 


\section{Acknowledgements}

We would like to acknowledge the contribution from the AT20G Survey team at CSIRO/ATNF, University of Sydney, and Padova-SISSA.

\section{References}

[1] E. Fomalont et al., Depolarization Silhouettes and the Filamentary Structure in the Radio Source Fornax A, ApJ Lett. 346, L17 (1989)

[2] A.C. Taylor et al., The radio source counts at $15 \mathrm{GHz}$ and their implications for cm-wave CMB imaging, MNRAS 327, 11-L4 (2001)

[3] E. Waldram, et al., 9C: a survey of radio sources at $15 \mathrm{GHz}$ with the Ryle Telescope, MNRAS, 342, 915 (2003)

[4] G. Hinshaw et al., Three-Year Wilkinson Microwave Anisotropy Probe (WMAP) Observations: Temperature Analysi, (submitted to ApJ, Astro-Ph/060345, 2006)

[5] R. Ricci et al., First results from the ATCA 18 GHz pilot survey, MNRAS, 354, 305 (2004)

[6] P.P. Roberts, M.R. Leach, W.E. Wilson, An $8 \mathrm{GHz}$ Bandwidth Analogue Correlator System for the Australian Telescope Compact Array (in preparation 2006)

[7] K.Y. Lo, et al., AMiBA: Array for Microwave Background Anistropy, in 20th Texas Symposium on Relativistic Astrophysics, 2000, eds., J.C. Wheeler, H. Martel, AIP, Conf. Proc., 586, 172 (2001)

[8] E.M. Sadler et al., The properties of extragalactic radio sources selected at $20 \mathrm{GHz}$, (submitted to MNRAS, Astro-Ph/0603437, 2006)

[9] e Zotti et al., Predictions for high-frequency radio surveys of extragalactic sources, A\&A, 431, 893, (2005)

[10] C.L. Bennett et al., First-Year Wilkinson Microwave Anisotropy Probe (WMAP) Observations: Foreground Emission, ApJS, 148, 97 (2003)

[11] M. Tucci et al., Predictions on the high-frequency polarization properties of extragalactic radio sources and implications for polarization measurements of the cosmic microwave background, MNRAS, 349, 1267 (2004)

[12] R. Ricci et al., High-frequency polarization properties of southern Kühr sources, A\&A, 415, 549 (2004)

[13] J.A. Peacock, The high-redshift evolution of radio galaxies and quasars, MNRAS, 217, 601 (1985)

[14]F.N. Owen, S.R. Spangler \& W.D. Cotton, Simultaneous radio spectra of sources with strong millimeter components, AJ, 85, 3510 (1980)

[15] S.J. Tingay et al., ATCA Monitoring Observations of 202 Compact Radio Sources in Support of the VSOP AGN Survey, PASJ, 55, 351 (2003)

[16] A. Ciaramella et al., A multifrequency analysis of radio variability of blazers, A\&A, 419, 485 (2004)

[17] R. Barvainis et al., Radio Variability of Radio-quiet and Radio-loud Quasars, ApJ, 618, 108 (2005)

[18] Valtaoja et al., Five years monitoring of extragalactic radio sources. II - Quiescent spectra and the evolution of outbursts, A\&A, 203, 1 (1988) 